Wie regionale Kooperation eine nachhaltige Wirtschaftsweise fördert

\title{
Vom Korn zum Brot in der Region
}

\author{
Betriebe können zu einer nachhaltigen Regionalentwicklung beitragen, indem \\ sie mit anderen Unternehmen und Akteuren einer Region kooperieren. Insbe- \\ sondere im Ernährungsbereich kann regionale Kooperation dazu beitragen, ex- \\ terne Effekte zu internalisieren, Informations- und Glaubwürdigkeitsdefizite zu \\ überwinden, Investitionsrisiken für den Einzelnen zu reduzieren und die Markt- \\ position der Partner zu stärken. Dies wird an Beispielen illustriert.
}

$\mathrm{L}$

Von Anke Witzel und Martin Tischer andauf landab gründen sich in den letzten Jahren Initiativen zur regionalen Herstellung und Vermarktung von Produkten, insbesondere von Lebensmitteln. Diese Initiativen werden in den Zusammenhang mit nachhaltiger Regionalentwicklung gestellt, weil sie

- Transporte und Stoffströme reduzieren,

- regionale Arbeitsplätze schaffen oder sichern,

- die Wertschöpfung in vom Strukturwandel benachteiligten ländlichen Räumen erhöhen,

- oft mit ökologischen Produktionsstandards einher gehen,

- traditionelle, regionstypische Anbau- oder Verarbeitungsmethoden stabilisieren bzw. wiederbeleben und

- das Bewusstsein der Bevölkerung für Herkunft und Produktionsweise von Lebensmitteln schärfen.

\section{Thesen}

In der Realität gibt es zahlreiche regionale Initiativen, die wirtschaftlich tragfähig sind und gleichzeitig ökologische und soziale Ziele verfolgen und erreichen. Interessanterweise handelt es sich meist nicht um einzelbetriebliche Initiativen. Betriebe und weitere Akteure kooperieren bei der Herstellung und Vermarktung von Produkten aus der Region für die Region (1).

Kooperation wird dabei verstanden als - neben Markt und Hierarchie - eine von drei idealtypischen Koordinationsformen für wirtschaftliche Austauschprozesse (2), bei der nicht Preise oder Weisungen, sondern Verhandlungen und Argumente den Leistungsaustausch koordinieren. Kooperation ist ein Weg, strukturelle Barrieren gegenüber einer nachhaltigen Entwicklung zu überwinden. Vielfach hängen diese mit der Unvollkommenheit des Marktes zusammen (3). Eine nachhaltige Entwicklung wird behindert durch:
- Markt- und Staatsversagen bei der Internalisierung externer Effekte,

- Informations- und Glaubwürdigkeitsdefizite hinsichtlich ökologischer und sozialer Produktqualitäten,

- ein erhöhtes Investitionsrisiko in neuen Marktfeldern sowie

- die in vielen Fällen vergleichsweise geringe Schlagkraft und Größe regionaler Unternehmen auf globalisierten Märkten.

Die folgenden Argumente für Unternehmenskooperationen beruhen auf unterschiedlichen Theorieansätzen für das Bestehen der Barrieren und werden mit Beispielen von regionalen Kooperationen belegt.

\section{- Internalisierung externer Effekte}

Allgemein gilt, dass nachhaltige Produkte aufgrund von höheren Kosten einer umweltverträglichen Herstellung teurer sind. Der Preis von ökologisch produzierten Lebensmitteln liegt beispielsweise über demjenigen vergleichbarer konventioneller Produkte. Denn die Preise sagen nicht die ökologische Wahrheit, die Hersteller müssen selten die negativen Effekte ihrer Produktionsweise, zum Beispiel die Belastung von Gewässern durch Überdüngung, in ihre Kostenrechnung einbeziehen. Unternehmen, die gemeinsam neue ökologische oder sozialverträgliche Rahmenbedingungen innerhalb eines Teilmarktes definieren, können damit zur Internalisierung dieser externen Effekte beitragen. Ausdruck dieser Rahmenbedingungen sind Produktionskriterien oder Richtlinien, die von den meisten regionalen Initiativen formuliert und überprüft werden. Häufig werden für die landwirtschaftliche Produktion die bekannten Richtlinien der Arbeitsgemeinschaft für ökologischen Landbau (AGÖL) übernommen (TAGWERK Genossenschaft in Dorfen, Hermannsdorfer Landwerkstätten in Glonn) (4). Bei BRUCKER LAND sind für jedes Produkt spezifische Kriterien zwischen Erzeugern, Handwerk und Verbrauchern ausgehandelt worden (5).

\section{- Verringerung von Informations- und Glaubwürdigkeitsdefiziten}

Nachhaltige Produkte haben eine neue, besondere Qualität. Diese muss bei der Vermarktung glaubwürdig herausgestellt werden. Gerade auch der höhere Preis der regionalen Lebensmittel macht einen erhöhten Informations- und Kommunikationsaufwand erforderlich. Unternehmen, die gemeinsam vermarkten oder mit bekannten Glaubwürdigkeitsträgern wie Verbänden des ökologischen Landbaus verbunden werden, können die einzelbetrieblichen Kosten für die Informationsvermittlung und die Marketingmaßnahmen senken. Auch in diesem Zusammenhang sind transparente Richtlinien (siehe oben) ein wichtiges Instrument.

Die Initiative Natur und Leben Bregenzerwald wurde 1992 mit dem Ziel gemeinsamer Vermarktungsaktivitäten von Gastwirten und Landwirten aus der Region gegründet. Es werden gemeinsam Märkte, Info-Veranstaltungen, Hofläden und Werbung betrieben. Der Heimat- und Verkehrsverband Eichsfeld e.V. kontrolliert bei Eichsfeld pur die Einhaltung der offensiv vermarkteten Richtlinien.

\section{Reduzierung des Investitionsrisikos}

Die Entwicklung innovativer Produkte und Produktionsweisen - und solche stellen nachhaltige Produkte dar - ist mit hohen Risiken verbunden. Risiken entstehen insbesondere, wenn die (künftige) Produktion mit Investitionen verbunden ist, die nicht oder nur in geringem Maße für alternative Zwecke nutzbar sind (sogenannte partnerspezifische Investitionen). Die Entscheidung für eine Umstellung der Werbung, die Anschaffung einer Maschine oder auch die Schulung des Personals für die Herstellung und Vermarktung eines neuen Produktes ist sicherer, wenn sich das entsprechende Unternehmen auf die Zulieferung der benötigten Vorleistungen und die Abnahme der eigenen Produkte verlassen kann. In solchen Fällen können längerfristige Kooperationen mit in der Produktionskette vor- und nachgelagerten Betrieben zur Verteilung des Risikos auf mehrere Betriebe und damit zur Risikominimierung für den Einzelbetrieb führen. Unternehmenskooperation kann somit die Umsetzung von Innovationen im Sinne einer nachhaltigen Entwicklung ermöglichen, bei 
denen das Risiko für ein einzelnes Unternehmen aufgrund notwendiger partnerspezifischer Investitionen zu hoch wäre.

Bei einer größeren Zahl von Projekten ist die Verringerung von Unsicherheiten durch partnerspezifische Investitionen zumindest ein Zusatznutzen der Kooperation. Werden wie bei Eichsfeld pur oder BRUCKER LAND Investitionen in die Wiederbelebung regionaler Infrastrukturen, etwa von Schlachthäusern geplant, muss eine entsprechende Zulieferung bzw. Nutzung von vornherein sichergestellt sein.

Die Marktgenossenschaft der Naturland-Bauern $e G$ in Lippetal-Lippborg macht langfristige Verträge mit den dort organisierten regionalen Bauern. Naturland wiederum sichert sich durch Abnahmegarantien der Famila Warenhaus GmbH \& Co. KG in Soest ab. So konnten innerhalb von wenigen Jahren zahlreiche Landwirte auf ökologischen Landbau umstellen, um mittlerweile 57 Produkte regional ökologisch anzubieten (Stand 1999).

\section{Stärkung der Marktposition}

Last but not least können nachhaltige Produkte um so besser produziert und vermarktet werden, je stärker die Position am Markt ist. Unternehmenskooperation stellt damit für viele klein- und mittelständische Betriebe eine Strategie dar, um Größenvorteile und Komplementaritäten auszunutzen und ökonomisch erfolgreicher zu sein. Die getrennte Verarbeitung und Veredelung re- gionaler Rohstoffe durch Mühlen, Schlachthäuser, Molkereien usw. lohnt erst ab einer bestimmten Größenordnung. Der preisgekrönte Schlachthof in Überlingen, die Getreidemühle in Fürstenfeldbruck oder die Upländer Bauernmolkere $i$ in Usseln sind Beispiele dafür. Im Bregenzer Wald gelang durch die Kooperation von 191 Betrieben aus Landwirtschaft, Tourismus und Öffentlichkeit (Stand Juni 1998) eine Profilierung als internationale Käseregion.

\section{- Erfolgsfaktoren für eine nachhal- tige Regionalentwicklung}

Die Beispiele zeigen, dass Kooperation auf unterschiedliche Weise den Weg zu einem nachhaltigen regionalen Wirtschaften ebnen kann. Es wird immer wieder darauf hingewiesen, dass die Region ein Umfeld bietet, das den Aufbau von Vertrauen zwischen den potenziellen Kooperationspartnern erleichtert. Außerdem sind regionale Qualitätsprodukte gerade im Ernährungsbereich für einige Produktlinien ein vielversprechendes neues Marktsegment. Dies führt dazu, dass immer mehr Unternehmen regionalen Kooperationen aufgeschlossen gegenüberstehen.

Noch wichtiger scheint es jedoch, dass sich auf regionaler Ebene sehr oft Träger und Initiatoren für Unternehmenskooperationen finden, die aufgrund von Verantwortungsgefuihl für die Entwicklung ihrer Region sowie emotionaler Nähe zu ihrer Heimat ein solches Engagement entwickeln.

\section{Abb.: Die Organisation der Initiative BRUCKER LAND}

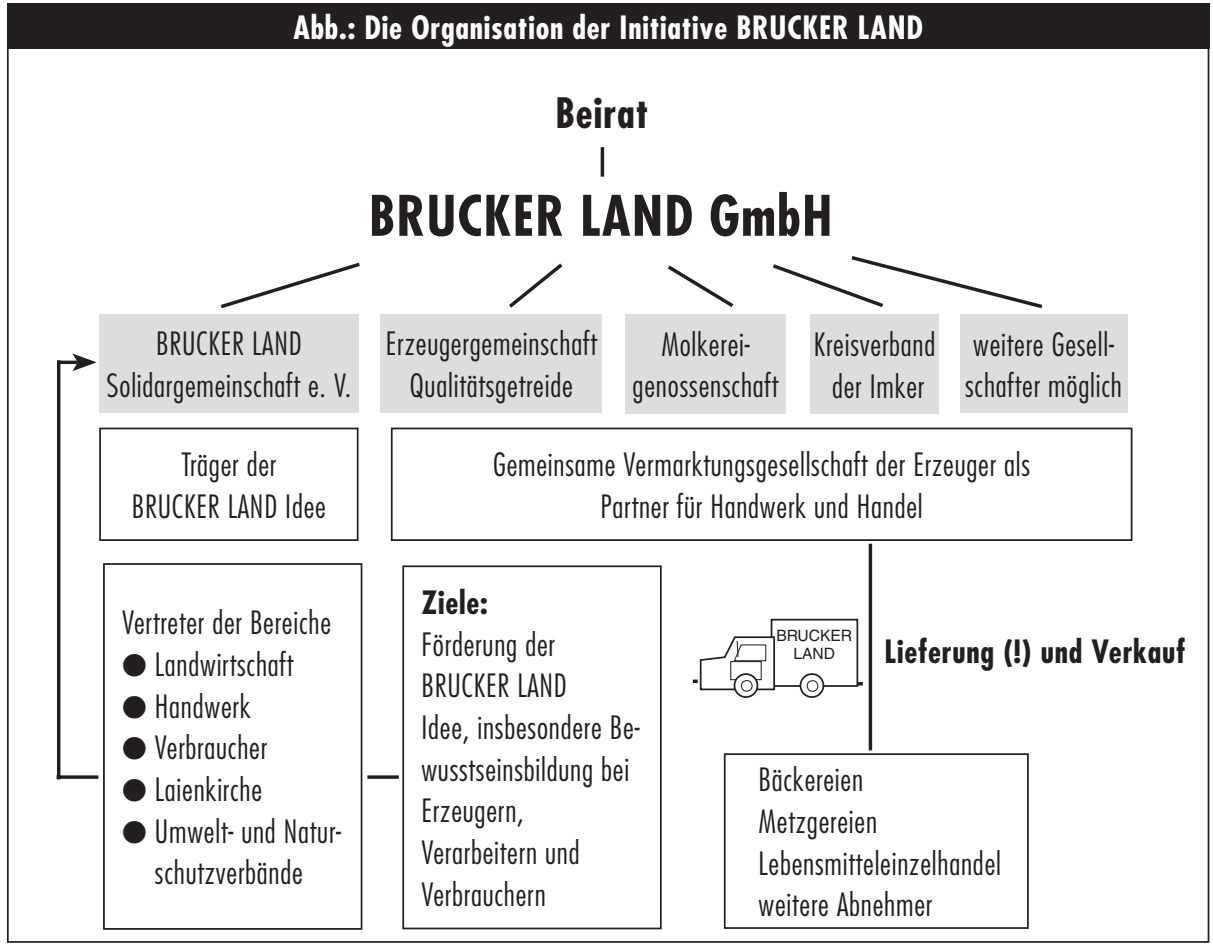

Als Initiatoren- und Trägerorganisationen fungieren sehr unterschiedliche Organisationen mit staatlichem, kirchlichem, wissenschaftlichem oder NGOHintergrund. Diesen kommt in zweifacher Hinsicht eine Schlüsselrolle zu. Zum einen initiieren und vermitteln sie Kooperationen und stabilisieren diese. Zum anderen sind sie entscheidend dafür verantwortlich, dass mit den Unternehmenskooperationen ein Beitrag zu einer nachbaltigen Regionalentwicklung geleistet wird. Denn in den meisten Beispielen stellen die Initiatoren- und Trägerorganisationen sicher, dass neben ökonomischen auch ökologische und soziale Ziele angestrebt und realisiert werden. Diese Kombination von gemeinwohlorientierten Akteuren mit ,eigennützigen“ Unternehmen, die durch eine entsprechende Organisationsstruktur institutionalisiert werden sollte (vergleiche das Beispiel BRUCKER LAND in der Abbildung), scheint ein tragfähiges Modell für zukunftsfähiges regionales Wirtschaften in vielen Bereichen zu sein.

\section{Anmerkungen}

(1) Der Aufsatz beruht auf Erfahrungen der Autorlnnen aus ihrer wissenschaftlichen Beratungstätigkeit. Vgl. u.a. Tischer, M.: Unternehmenskooperation und nachhaltige Entwicklung in der Region (erscheint demnächst); Peters, U./ Sauerborn, K./Spehl, H./ Tischer, M./ Witzel, A.: Nachhaltige Regionalentwicklung - ein neues Leitbild für eine veränderte Struktur- und Regionalpolitik Trier (NARET), 1996. (2) Vgl. z.B. Minsch, J. u.a.: Mut zum ökologischen Umbau: Innovationsstrategien für Unternehmen, Politik und Akteurnetze. Basel 1996; Dörsam, P./ Icks, A.: Vom Einzelunternehmen zum regionalen Netzwerk: Eine Option für mittelständische Unternehmen. Stuttgart 1997.

(3) Vgl. Schneidewind, U.: Die Unternehmung als strukturpolitischer Akteur. Marburg 1998.

(4) Die im Rahmen dieses Artikel aus Platzgründen jeweils nur kurz angesprochenen Initiativen können zum Großteil in folgenden Dokumentationen genauer nachgelesen werden: http://www.municipia.at/, http://www.reginet. de/, http://www.zukunftsregionen.de/

(5) Karg, L./ Schindelmann, P.: Die Brucker Land Solidargemeinschaft. In: Mayer-Ries, J. (Hrsg.): Zwischen globalen und lokalen Interessen. Loccum 1998, S. 247-255.

\section{Die Autorlnnen}

Anke Witzel und Martin Tischer sind bei B.A.U.M. Consult, München verantwortlich für den Bereich Nachhaltige Regionalentwicklung.

Kontakt: Thalkirchner Str. 180, 81371 München, Tel. 089/ 767368-26, Fax 089/7250807, E-mail: witzel@baum-consult.de, tischer@baum-consult.de 
(c) 20I0 Authors; licensee IÖW and oekom verlag. This is an article distributed under the terms of the Creative Commons Attribution Non-Commercial No Derivates License (http://creativecommons.org/licenses/by-nc-nd/3.o/), which permits unrestricted use, distribution, and reproduction in any medium, provided the original work is properly cited. 